\title{
TERRITORIOS EN RIESGO HÍDRICO: PROYECTO TERRITORIAL Y MULTIESCALARIDAD \\ Caso: Gran La Plata, Provincia de Buenos Aires, Argentina
}

Territories in water risk: territorial project and multiscale

Case: Gran La Plata, Province of Buenos Aires, Argentina

\section{López, Isabel;}

Centro de Investigaciones Urbanas y Territoriales, Facultad de arquitectura y urbanismo, De la Universidad Nacional de La Plata. Email: ilopez.arqui@gmail.com

Fisch, Sara;

Centro de Investigaciones Urbanas y Territoriales, Facultad de arquitectura y urbanismo, De la Universidad Nacional de La Plata. Email: fischsara@yahoo.com.ar

\section{Giusso, Cecilia;}

Centro de Investigaciones Urbanas y Territoriales, Facultad de arquitectura y urbanismo, De la Universidad Nacional de La Plata. Email: ceciliagiusso@fibertel.com.ar

Carluccio, Braian;

Centro de Investigaciones Urbanas y Territoriales, Facultad de arquitectura y urbanismo, De la Universidad Nacional de La Plata. Email: braiancarluccio@hotmail.com

\section{RESUMEN}

Para abordar la problemática del riesgo hídrico por inundación se considera necesario trabajar en las diferentes escalas afectadas. Por ello, el caso que se desarrolla en el Gran La Plata, Provincia de Buenos Aires, Argentina, toma como unidad de análisis la región en su totalidad, la sub cuenca del Arroyo el Gato como área de verificación de la situación de riesgo y de proyecto territorial a largo plazo (medidas no estructurales). Y, por último, se presenta como parte del subsistema de medidas estructurales o de obras, un proyecto urbanístico y arquitectónico que actuará en la interfase entre problemáticas ambientales y sociales en un barrio de la sub cuenca del arroyo Pérez, afluente Del Gato. Todo surge de dos Proyectos de Investigación ${ }^{1}$.

Palabras clave: Riesgo Hídrico, Proyecto Territorial, Multiescalaridad, Sensibilización Social Y Ambiental Bloque temático: Análisis y proyecto territorial

\begin{abstract}
To address the problem of water risk due to flooding, it is considered necessary to work on the different scales affected. For this reason, the case that takes place in Gran La Plata, Province of Buenos Aires, Argentina, takes as a unit of analysis the region as a whole, the sub-basin of Arroyo del Gato as an area of verification of the risk situation and of the long-term territorial project (non-structural measures). And finally, it is presented as part of the subsystem of structural measures or works, an urban and architectural project that will act in the inter phase between environmental and social problems in a neighborhood of the basin of the Perez stream, Del Gato tributary. Everything arises from two Research Projects.
\end{abstract}

Keywords: Water Risk, Territorial Project, Multiscale, Social and Environmental Sensitization Topic: Analysis and territorial project

\footnotetext{
${ }^{1}$ 2014-2017 Proyecto "TERRITORIOS VULNERABLES Y PAISAJES EMERGENTES. Estrategias de gestión para su transformación. Caso: Gran La Plata". UNLP. Directora: Isabel López; Codirector: Juan Carlos Etulain

2014/16 Proyecto: "LAS INUNDACIONES EN LA PLATA, BERISSO Y ENSENADA: ANÁLISIS DE RIESGOS, ESTRATEGIAS DE INTERVENCIÓN. HACIA LA CONSTRUCCIÓN DE UN OBSERVATORIO AMBIENTAL". UNLP-CONICET. Directora: Alicia Ronco; Codirector: Isabel López
} 


\section{Introducción}

El objetivo de la ponencia es presentar la interrelación escalar que mantienen los proyectos territoriales para tratar de solucionar los problemas de riesgo hídrico por inundación en el Gran La Plata (RMBA - RGLP-Figura $N^{\circ} 1$ ) realizado a través de un proyecto de investigación. Para ello, se realizó la cartografía de riesgo; se idearon lineamientos que deberían conducir el ordenamiento urbano y territorial para el Gran La Plata y la sub cuenca del Gato con los cuales podrían programarse simultáneamente acciones y regulaciones.

También se presenta -especialmente- y para seguir la lógica de lo interescalar las obras (medidas estructurales) de la sub cuenca del arroyo Del Gato y las Áreas de Retención Temporal de Excedentes Hídricos (ARTEH) propuestas como partes del proyecto con el objeto de incorporarlos al sistema urbanístico suroeste de La Plata (cuenca alta del Gato) como subsistema de Parques del Agua para prevención de inundaciones y optimizar el funcionamiento del ciclo del agua. A su vez, la propuesta urbanística y arquitectónica con Equipamientos Comunitarios Ambientales (ECAs) multifuncionales que actúen como puntos de referencia de la población barrial y contribuyan a la construcción de resiliencia social, especialmente para el barrio del Retiro. La estrategia metodológica se basa en el estudio del caso GLP, identificando los paisajes emergentes en los territorios vulnerables con riesgo de inundación considerando las cuencas y subcuencas como unidades de análisis e intervención. Esta estrategia "comparte la idea de un caso considerado de interés en sí mismo, y abordado en toda su complejidad. Tiene una fuerte orientación interpretativa. Su propósito es analizar los procesos y fenómenos sociales, prácticas, instituciones y patrones de comportamiento para desentrañar los significados construidos alrededor de ellos, en un contexto o entorno que puede ser de redes de relaciones sociales y ambientales" (Sautu 2003: 78-83)

Se emplearon instrumentos técnicos, como Sistemas de Información Geográfica para la Cartografía de Riesgo y relevamientos de campo; el estudio de casuísticas; de participación social, de funcionarios relacionados al tema, de técnicos de otras disciplinas; y de organizaciones barriales para elaborar los programas de necesidades y actividades; y proyectuales, basados en hipótesis que permitan indagar diferentes aproximaciones para producir el conocimiento necesario.

\section{Aproximación a la problemática de las inundaciones en el Gran La Plata}

El Gran La Plata (RMBA-RGLP, Figura 1) como parte de toda región metropolitana, en este caso la de Buenos Aires, tiene como primera problemática las cuestiones interjurisdiccionales asociadas a la gestión en general, y a las problemáticas ambientales y de ordenamiento territorial en particular. Por lo tanto, la misma gestión del riesgo por lluvias extraordinarias se transforma en amenaza y la emergencia de territorios vulnerables en toda la región así como la elevación del nivel del río de La Plata en Berisso y Ensenada una cuestión problemática compleja.

El GLP tiene una extensión de 1.162 Km2 (116.200 Ha.) con $17.857 \mathrm{Ha}$. urbanizadas, 801.901 habitantes, es parte del Litoral Sur Metropolitano de Buenos Aires y de la cuenca de la selva sur. Son parte de ella tres Partidos junto a la jurisdicción del Puerto La Plata. Los Partidos son: Ensenada de 101 km² (10.100 Ha), 1878 Ha urbanas y 58.693 habitantes; Berisso de 135 km², (135.000 Ha.) $1672 \mathrm{Ha}$ urbanas y 89.096 habitantes y La Plata con $926 \mathrm{~km}^{2}(92.600 \mathrm{Ha}), 14.307 \mathrm{Ha}$ urbanas y 654.112 habitantes.

Como parte de un asentamiento "litoral", que aloja en general actividades incompatibles que compiten por el uso de los recursos suelo y agua, constituye un territorio de interfase, reconocido como frágil a partir de la ocupación de las planicies de inundación del río en Berisso y Ensenada, con problemas de inundaciones cíclicas, impacto negativo de las actividades industriales y del área de enterramiento de residuos sólidos. El Partido de La Plata, que ocupa parte de la pampa ondulada interior, se destaca por su Casco Fundacional, y por la fertilidad de sus tierras, que mantienen históricamente una producción hortícola y florícola importante, que le otorga identidad, aunque el crecimiento urbano por extensión, la está debilitando. 
En este proceso de crecimiento los diez (10) arroyos que nacen en La Plata (cuenca alta), atraviesan el área urbana (cuenca media) y llegan al Río de la Plata a través de los bañados (cuenca baja) con dirección surestenoreste fueron pasando de asumir el rol de drenajes o desagües urbanos o evacuación de efluentes industriales, a ser causa de inundaciones, porque las subcuencas no fueron tratadas con la lógica de ocupación que deberían haber tenido. Hoy cada uno de ellos divaga en la subcuenca de pertenencia, conformando un sistema de drenaje autónomo -en su mayoría- entubados en una gran parte, y otros a través de sus cauces naturales, pero muy degradados, a veces muy sucios y canalizados para sortear el Bañado de Maldonado. Esta última situación resulta una gran restricción para el libre escurrimiento del agua porque una planicie de configuración geológica aluvional (planicie de inundación del Río de la Plata) casi no tiene pendiente en un ancho de aproximadamente $9 \mathrm{~km}$. Esta particularidad se constituye en una limitación importante para cualquier asentamiento humano, porque tiene suelos arcillosos, anegamientos permanentes y la napa freática salinizada, cerca de la superficie.

Sobre este estado ambiental de base, se suma el cambio climático, que se hizo presente en varias ocasiones en forma de lluvias extraordinarias e inundaciones $(2002,2008)$ hasta el desastre del 2 de abril de 2013, con una precipitación extraordinaria de $400 \mathrm{~mm}$, lloviendo $313 \mathrm{~mm}$ en 6 horas, cuando lo ordinario para el mes de abril es $111 \mathrm{~mm}$, dejando un resultado catastrófico, se registraron 89 muertes. Los registros demuestran que desde las primeras décadas del siglo XX existen inundaciones por lluvia y desborde de los arroyos Carnaval, Martin, Rodríguez, Don Carlos, del Gato, Maldonado, del Pescado y otros afluentes menores, pero lluvias e inundaciones de los años 2002 y 2008 no llamaron a la alerta necesaria. Por último, y para terminar con la caracterización de las amenazas e inundaciones en la región es necesario mencionar que las sudestadas del Río de La Plata hostigan Ensenada y Berisso desde sus fundaciones, aunque estos eventos se pueden anticipar como fenómeno. Ambas amenazas es necesario preverlas y difieren en su gestión. Para esta última existe una cultura de previsión para las inundaciones por lluvia, no.

Se puede decir entonces, que las inundaciones en la región son un fenómeno y un proceso de acontecimiento periódico, que puede resultar de tres factores, y que, en forma combinada, aumenta aún más el nivel de peligrosidad y riesgo, a saber: las precipitaciones por encima de la media y extraordinarias; napas freáticas que por saturación aumentan la presión hacia arriba a partir de su elevación, y la sudestada, que eleva el nivel del Río de la Plata e inunda el litoral de Ensenada y Berisso además de no permitir el libre escurrimiento de los arroyos. Esto combinado con una urbanización de llanura -en parte pampa ondulada-, atravesada por diez arroyos, constituyen a la sociedad asentada en sus bordes y planicies de inundación en vulnerables. 


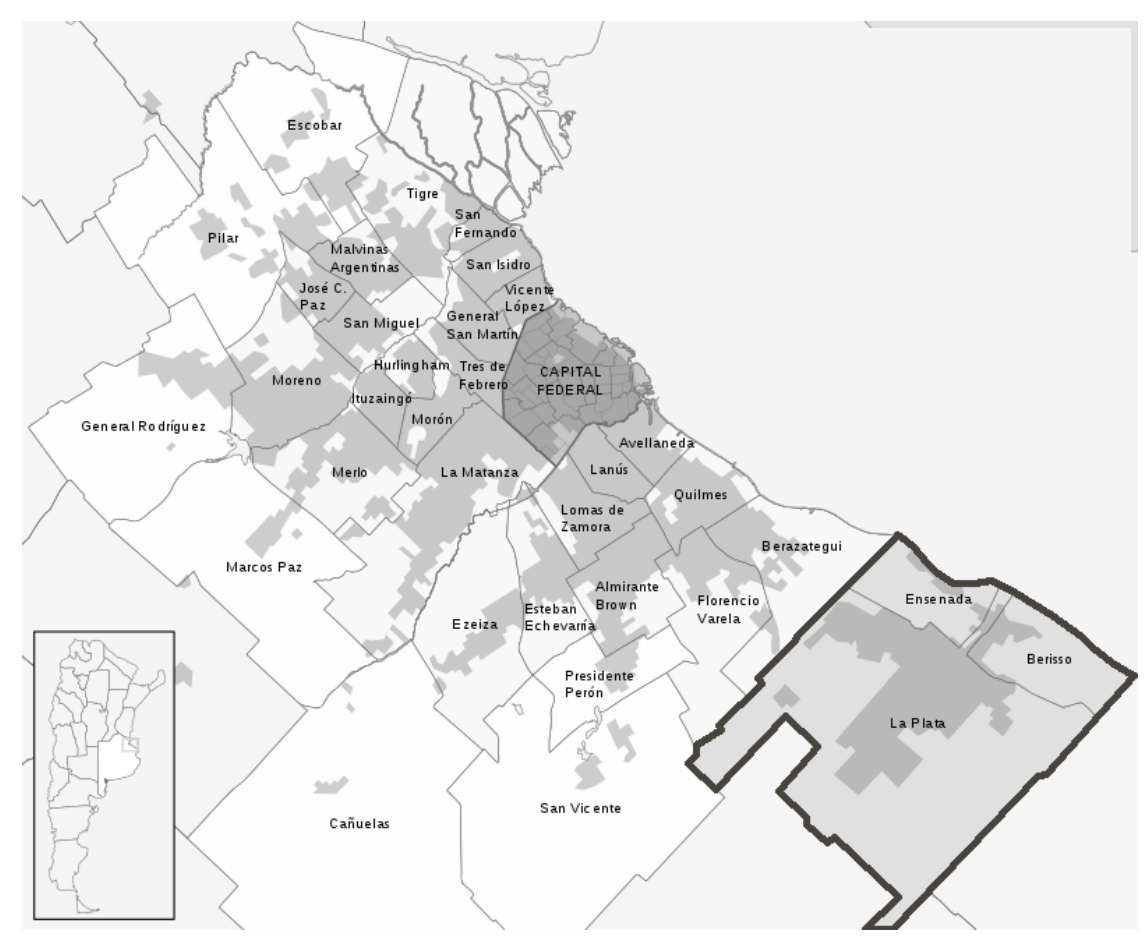

Figura 1. RMBA y GLP. Elaboración propia

\section{La Gestión Integral del Riesgo Hídrico por Inundaciones Urbanas (GIRHIU)}

El análisis de territorios vulnerables a escala metropolitana (atravesados por lógicas territoriales y ambientales) en el marco de políticas de reducción del riesgo hídrico por inundación, no ha sido suficientemente explorado en la investigación metropolitana, y en particular, en aquellas investigaciones orientadas hacia la formulación de modelos de adaptación y/o mitigación en territorios pampeano-litorales como el caso de estudio.

Claudia Natenzon (1995) reflexionando acerca del riesgo dice que éste existe cuando es posible cuantificarlo; cuando el riesgo no es cuantificable se transforma en incertidumbre (Funtowicz, 1994) y, en problemáticas muy complejas, como desastres naturales. La Incertidumbre se relaciona con el desconocimiento científico sobre la materia, los valores que se están poniendo en juego y, lo que se arriesga, en la toma de decisiones (Funtowicz y Ravetz, 1993).

Por lo tanto, desde lo conceptual, se reconoce la necesidad de aplicar la teoría social del riesgo. Pero para la modelización de la situación de riesgos y su análisis -desde lo empírico- se utilizó la metodología propuesta por Ribera Masgrau (2004), donde la vulnerabilidad se reemplaza conceptualmente -dentro del componente humano- por la exposición más la susceptibilidad, a partir de la aplicación de la siguiente fórmula:

\section{Riesgo hídrico = Amenaza $\times$ Vulnerabilidad (Exposición + Susceptibilidad) $/$ Resiliencia}

El riesgo de inundación es producto de la interrelación de amenazas y vulnerabilidades es una construcción social, dinámica y cambiante, diferenciada en términos territoriales y sociales. (Allan Lavel, 1997)

En definitiva, metodológicamente se buscó la construcción de un modelo que represente adecuadamente la realidad (aunque lógicamente la simplifique) y para ello se trabajó a partir de las siguientes dimensiones o variables que describen la vulnerabilidad urbana frente a la amenaza de inundaciones ante precipitaciones extraordinarias:

- Riesgo hace referencia a la probabilidad de que una población o parte de la misma, le ocurra algo a partir de una amenaza o peligro, nocivo o dañino, en este caso sufra inundación. 
- Amenaza o peligro se entiende como la posibilidad de ocurrencia de un evento físico proveniente -en este caso de la naturaleza- que puede causar algún tipo de daño a la sociedad teniendo en cuenta el deslizamiento y la retención de agua que puede causar la geomorfología.

- Exposición queda definida por la población, las propiedades, los sistemas u otros elementos presentes en las zonas donde existe la posibilidad que se produzca el evento que amenazante o peligroso (Naciones Unidas, 2009). La medida del grado de exposición puede incluir la cantidad de personas o los tipos de bienes en una zona o territorio.

- Susceptibilidad es el grado de fragilidad interna de un sujeto/s, objeto o sistema para recibir un posible impacto debido a la concurrencia de un evento adverso o amenaza (CIIFEN, 2013). Describe la precondición antes de sufrir un daño debido a las condiciones residenciales precarias, una infraestructura inadecuada, falta de acceso a los servicios básicos o con materiales de construcción "inestables" (Welz-Krellenberg, 2016). Pueden ser de naturaleza socioeconómico, material o física y ambiental, entre otras.

- Ambas, exposición y susceptibilidad conforman la vulnerabilidad, y expresa los niveles o grados de desequilibrio o desajuste entre la estructura social y el medio natural y construido. No puede tener valor absoluto, sino que su expresión es relativa. (Herzer y Gurevich, 1996)

Resiliencia se refiere a la capacidad de recuperación/respuesta para afrontar el impacto de un evento y/o estar prevenido para enfrentar un fenómeno adverso. Para la adaptación y prevención entonces es tan importante la relación tiempo-estrategia como la necesidad de aplicar medidas interescalares. El corto plazo tiene relación directa con el plan de contingencia, el mediano y largo plazo con las obras y el ordenamiento territorial como técnicas y guía para la adaptación, la mitigación y la prevención.

\section{Riesgo en el GLP y la Sub cuenca del Gato}

El resultado del análisis y la modelización de la amenaza y los grados de peligrosidad resultante además de la interrelación de las susceptibilidades y la exposición -la vulnerabilidad-permitieron obtener escenarios de riesgos acotados, con sus correspondientes mapas de riesgo de daños por inundación conformados por parámetros definidos en las distintas escalas abordadas. (Figura 2)

Estos mapas contribuyeron a reducir la incertidumbre y cuantificar por primera vez magnitudes que se desconocían, para actuar en consecuencia y seguir profundizando.

En este marco y analizando las relaciones entre indicadores del GLP y subcuenca Del Gato se pudo medir y relacionar magnitudes entre todas las subcuencas y la última, en cantidad de viviendas, habitantes, superficies urbanas, así como los respectivos niveles de riesgo.

Del total de población del GLP 801.901 habitantes, según el Censo Nacional de 2010, el 42, 89\% (343.987 habitantes) se localizan en la subcuenca Del Gato; y del total de superficie urbana con aproximadamente $17.857 \mathrm{Ha}$, el 28, 87\% $(5.157 \mathrm{Ha})$ pertenecen a la citada subcuenca. Del total de 305.969 viviendas del GLP según el Censo Nacional de 2010, el 49,73\% (152.175) se localizaban en la subcuenca o sea la mitad del patrimonio edilicio residencial.

Como tendencia se midió que entre 1996 y 2014, el crecimiento por extensión en la misma subcuenca alcanzó aproximadamente $27,5 \%$, quiere decir que desde la fundación de La Plata no ha dejado de ocuparse y que por lo tanto tiene edificios de diferentes momentos históricos, incluso muchos (especialmente en el centro) que han pasado por procesos de renovación. Proceso que a su vez aumentan la impermeabilización del suelo.

Del total de habitantes del GLP $420.976(49,17 \%)$ casi la mitad tienen niveles de riesgo de inundación muy alto y alto, mientras que casi un cuarto, el 23,72\%, pertenecen a la subcuenca Del Gato (Figura 2). 


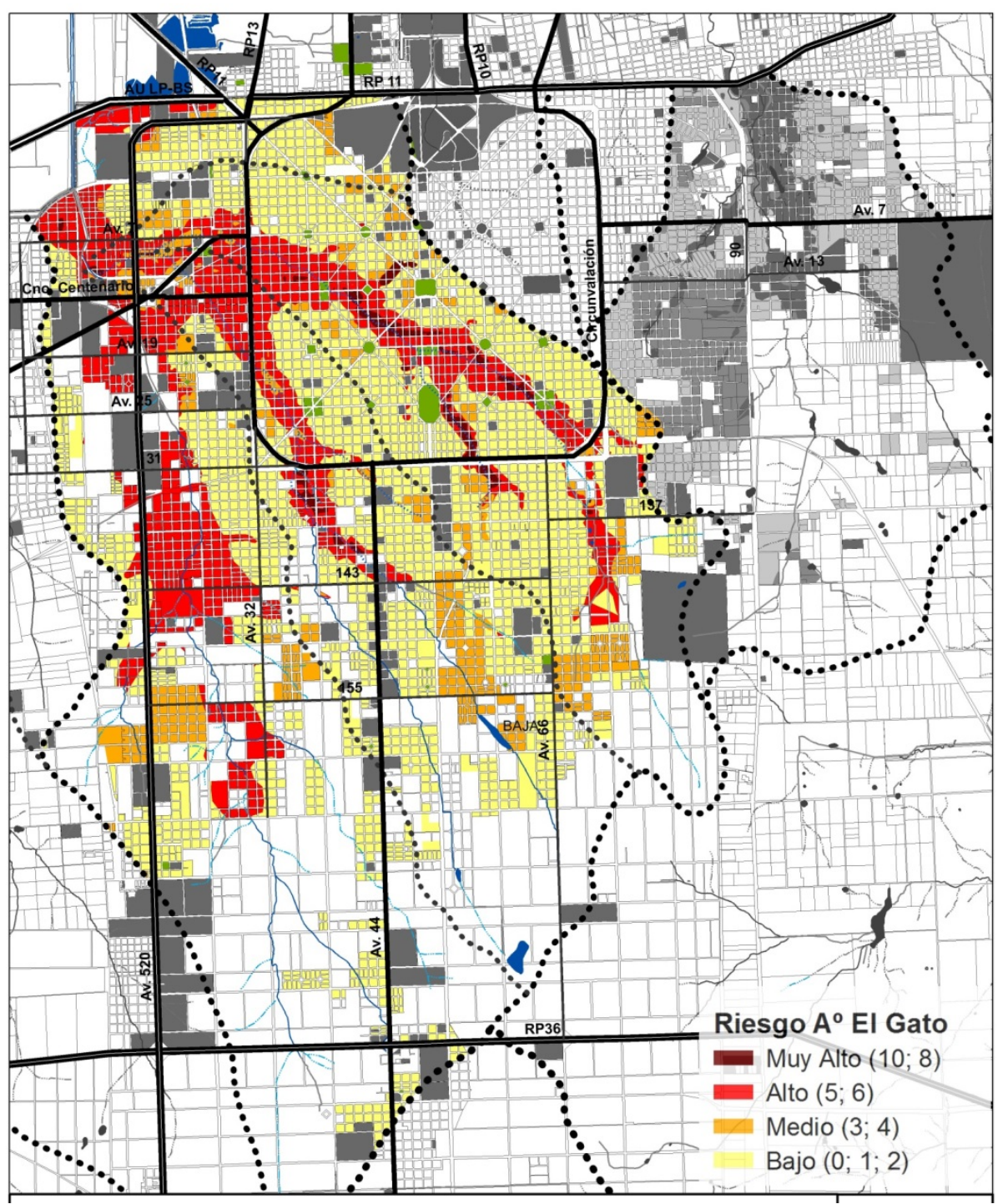

Figura 2. Niveles de Riesgo en la Subcuenca del Arroyo del Gato. Elaboración propia

\section{Las medidas estructurales y no estructurales como estrategias para la prevención y la adaptación. Escala de cuenca y subcuenca Arroyos del Gato y Pérez.}

Avanzar sobre la problemática de las inundaciones urbanas por lluvias extraordinarias implica que los estudios anteriores sirven como base para la formulación de planes, programas y proyectos en el marco de la gestión integral del riesgo. Pero la gestión del riesgo de inundación aun es una deuda pendiente, aunque sí se realizaron algunas obras.

Las prácticas y acciones orientadas a reducir el riesgo de desastres y sus efectos, así como también las consecuencias de las actividades relacionadas con el manejo de las emergencias y/o desastres comprenden acciones de prevención, mitigación y adaptación, así como la gestión de la emergencia y recuperación, 
denominadas medidas estructurales ${ }^{2}$ y no estructurales ${ }^{3}$ (DNGIRDRA, 2015). Los planes de contingencia (corto plazo) y de ordenamiento ambiental y territorial para la adaptación y prevención (largo plazo) aún esperan.

Paralelamente al desarrollo de este proyecto de investigación, el gobierno de la Provincia de Buenos Aires, como iniciativa política y a solicitud e insistencia de ciudadanos, asambleístas y movimientos sociales solo realizó obras de ingeniería, denominadas medidas estructurales, para la subcuenca del Gato iniciadas en 2014 y sin finalizar.

En la Figura 3 se reproduce las obras realizadas en la subcuenca del arroyo Del Gato, dimensionadas para tormentas de 10 años de ocurrencia y verificadas para R25 y R50 años, sin apreciar inconvenientes significativos. En ellas se identifican con el número 2 cinco Reservorios que la Dirección Provincial de Hidráulica, responsable del proyecto y su ejecución denomina Áreas de Retención Transitoria de Excedentes Hídricos (ARTEH).

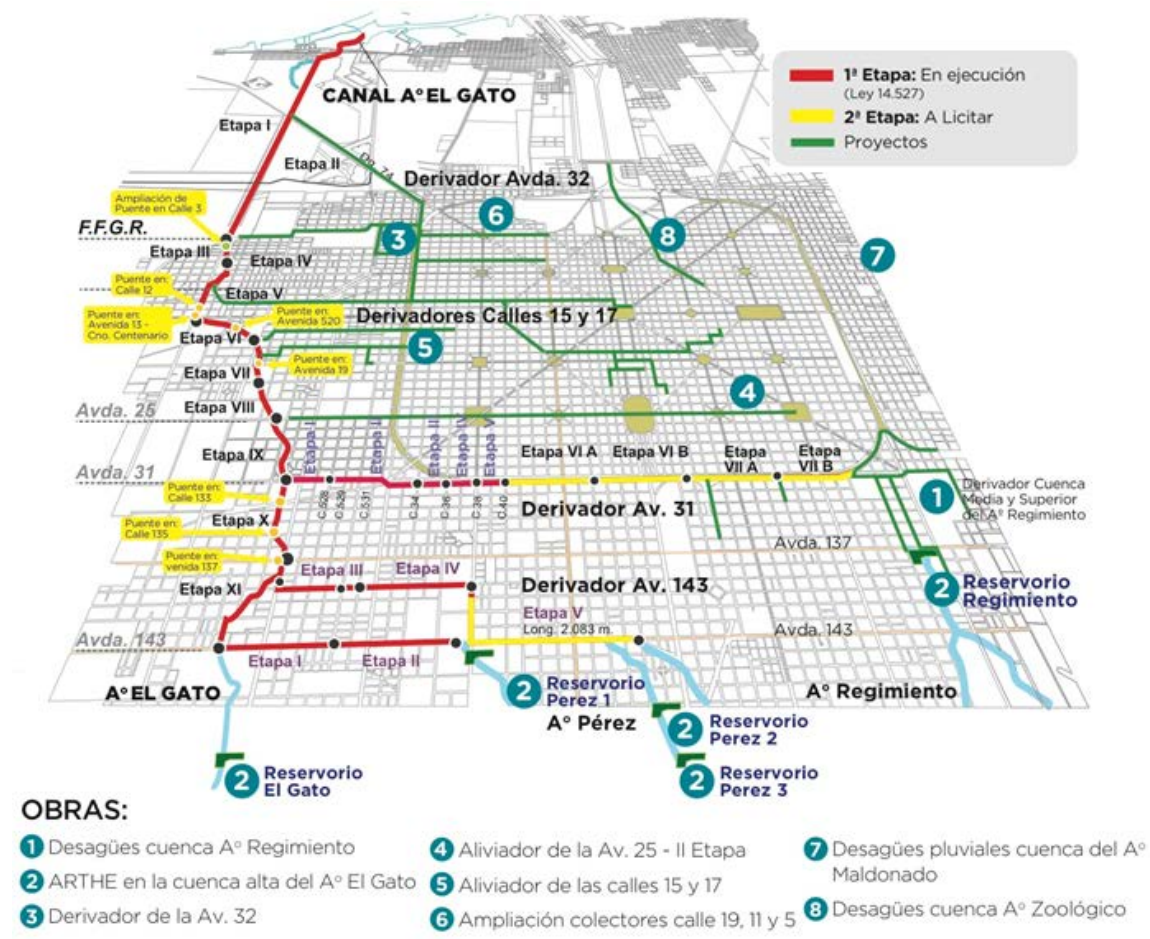

Figura 3: Plano de Obras para la Cuenca del Arroyo del Gato. Fuente: Municipalidad de La Plata

Las principales características que las definen son:

- La ampliación del tamaño de la sección transversal que acompaña el trazado del cauce del arroyo, proporcionalmente al grado del aporte recibido.

- Un revestimiento de hormigón armado para la sección del tramo medio con la construcción de taludes verticales para optimizar la sección hidráulica.

- La ampliación de la sección en el tramo bajo y de la desembocadura.

- La materialización de una rasante uniforme de canalización.

\footnotetext{
${ }^{2}$ Las medidas estructurales, se clasifican en "intensivas" y "extensivas". Si bien muchas de ellas demandan la intervención prioritaria de especialistas hidráulicos, las acciones vinculadas a la localización de reservorios y/o de control de escurrimiento para que éstas se concreten, requieren también recomendaciones de localización y previsión normativa en términos de ordenamiento territorial

${ }^{3}$ Las medidas "no estructurales" son las de ordenamiento territorial y la planificación y gestión que adoptan un carácter central si se está actuando con la intención de llevar a cabo "una gestión integral del riesgo de inundaciones". A su vez debe complementarse con los sistemas de alerta temprana y los planes de contingencia frente a ese tipo de eventos.
} 
- La limpieza de los cauces de los afluentes en el tramo alto, que de este a oeste se denominan arroyos: Regimiento, Pérez y del Gato, propiamente dicho.

- Por último, ejecutar cinco reservorios, denominados Áreas de Retención Temporaria de Excedentes Hídricos (ARTEH)

\subsection{Estrategias desde el Proyecto de Investigación.}

Desde el proyecto de investigación y de forma exploratoria -en base a la Cartografía de Riesgo realizada-y al análisis de otros casos, se idearon "medidas no estructurales" para el GLP y la Subcuenca del Gato. (Figura 4).

Sintéticamente se propone como lineamientos generales:

- Prohibir la expansión urbana sobre áreas de extrema vulnerabilidad ambiental (humedales) tanto en la cuenca alta como en la baja creando los sistemas municipales de áreas Protegidas.

- Conservar los bañados, los cauces de los arroyos abiertos y las cañadas que les dan origen como principales medios de drenaje natural con la divulgación de la necesidad de mantenerlos de esta forma.

- Adaptar la ocupación y el uso del suelo a la zonificación según los grados de riesgo hídrico de la sub cuenca con la participación de la comunidad afectada y programar las acciones y regulaciones para lograr grados óptimos de infiltración y drenaje.

- Orientar los crecimientos urbanos hacia lugares seguros (con medidas de promoción) programando simultáneamente las acciones y regulaciones.

- Recuperar áreas urbanas (por normativa) aun no ocupadas, como áreas rurales y espacio de infiltración

- Incorporar espacios de infiltración (en el total de las áreas urbanizadas y rurales) que colaboren con el funcionamiento del ciclo del agua (Precipitación = evapotranspiración + escorrentía + infiltración) en el marco de los atributos que tienen las cuencas hidrográficas como unidades territoriales de planificación y gestión de los recursos hídricos. Llevar a cabo políticas intensivas y demostrativas de infiltración generalizadas, para educar a conciudadanos y profesionales.

- Reestructurar los trazados y la subdivisión del suelo que limitan con los arroyos para prever el espacio público que deben proteger sus márgenes.

- Monitorear y gestionar el tratamiento de los márgenes de los arroyos de los drenajes y de las políticas de infiltración y arborización.

- Retardar la evacuación del flujo de las aguas pluviales, creando parques inundables en espacios apropiados a seleccionar, asociados a los cauces de los arroyos y/o adaptando parques existentes.

- Implementar la conformación de un Parque Agrario, a partir del límite de la RPN³6, que define el borde de crecimiento urbano de La Plata, con el propósito de contener la expansión urbana, y evitar de esta manera la perdida y mixtura de usos del borde periurbano de la región. Es un instrumento que promueve el desarrollo productivo local, incluyendo propuestas de desarrollo económico, social, recreativo y hasta turístico. En este sentido, se prevé desarrollar programas de recreación y ecoturismo, que incluyan las visitas de colegios a huertas comunitarias, huertas orgánicas que promuevan el uso racional de la tierra y cooperativas de productos locales y regionales. 


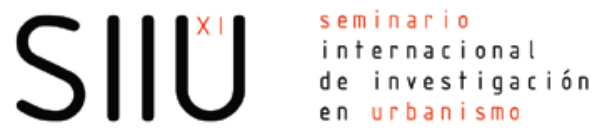
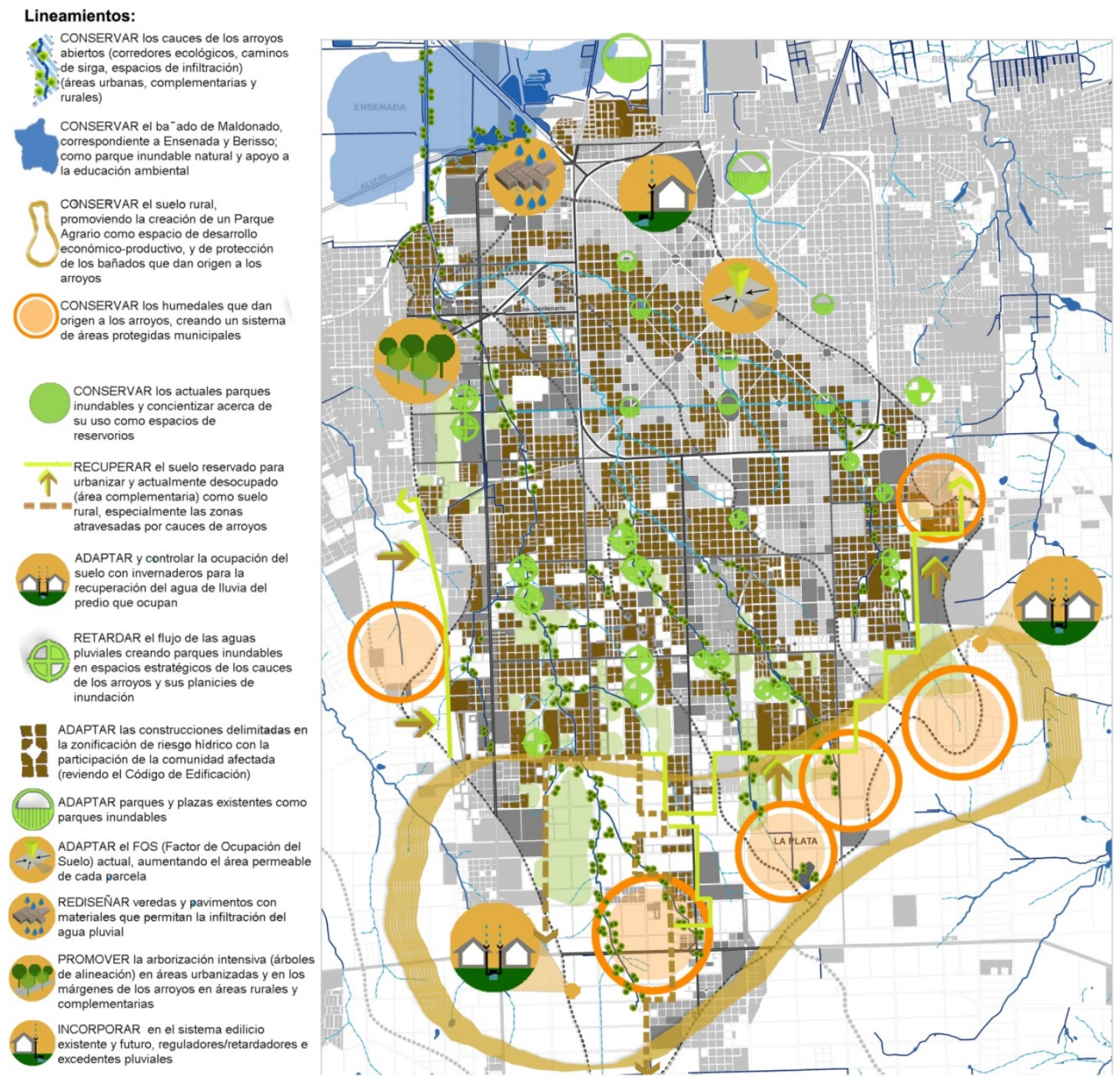

Figura 4: Lineamientos para prevención y adaptación Subcuenca del Gato. Elaboración propia

\subsection{Entre las necesidades barriales y las Áreas de Retención Temporal de Excedentes Hídricos. Barrio El Retiro.}

Como intervención de interfase y a escala de sector en un tramo de la Alta Cuenca, con el fin de atender la implementación del sistema de Áreas de Retención Temporaria de Excedentes Hídricos (ARTEH) que complementa las medidas estructurales definidas en el Plan Hídrico para la Sub Cuenca del Arroyo del Gato, se desarrolló como estrategia proyectual una propuesta urbanística de reestructuración que incorpora un Sistema de Parques de Agua (PA), acompañados a escala arquitectónica con Equipamientos Comunitarios Ambientales (ECAs).

En el barrio El Retiro, ubicado en la sub cuenca, y en coincidencia con uno de los ARTEH se presenta una propuesta urbanística que complementa las grandes obras y conforma una red o sistema de ARTEH y de barrios más amplio (Figuras 5 y 6 ). En todos los casos forman parte del proyecto estructural o subsistema en red, proponiendo y diseñando en cada uno, nuevos equipamientos de impacto barrial.

\subsection{Descripción del lugar}

Esta propuesta se desarrolló en el Barrio El Retiro, con aproximadamente 16.000 habitantes, y se caracteriza por la baja densidad (aprox.12 hab/ha.) y un $80 \%$ de la población conformada por niños y jóvenes. Presenta 
un alto grado de vulnerabilidad social, y prácticamente su totalidad se encuentra bajo la línea de pobreza; habiéndose multiplicado la presencia de asentamientos informales en los últimos años.

Dadas las características topo e hidrográficas del lugar, la población se encuentra asentada en una zona con alto riesgo de inundación. De hecho, en las ocurridas durante el año 2013, 420 familias fueron afectadas.

El lugar linda con zonas semirrurales, cuenta con calles de tierra, sin cloacas ni servicios de telefonía e internet, con la presencia del arroyo contaminado por residuos y desechos cloacales.

No posee espacios públicos ni equipamiento qué posibilite actividades para adolescentes (educación, oficios, recreación) y niños (escasez o ausencia de espacios de juego).

Cuenta con un Club de Barrio "Corazones de El Retiro" (160 entre 49 y 50) que oficia de condensador social y donde además desarrolla sus actividades el Centro Comunitario de Extensión Universitaria №3 de la Universidad Nacional de La Plata. Desde 1999 está constituido como Biblioteca Popular y lleva a cabo proyectos sociales, deportivos y culturales como fútbol infantil; comedor-merienda; copa de leche; centro de acopio y distribución de alimentos, artículos de limpieza y ropa en caso de inundaciones; ropero, centro de trueque y asamblea barrial, entre otros.

En los últimos diez años, la comunidad ha visibilizado la consolidación de su identidad como barrio a través de la presentación de proyectos en el presupuesto participativo municipal, los cuales fueron votados por todos los vecinos.

\subsection{Propuesta}

En el marco antes descripto, que incorpora un Sistema de Parques de Agua (PA), acompañados a escala arquitectónica con Equipamientos Comunitarios Ambientales (ECAs), se propone ${ }^{4}$ (figuras 5 y 6 ):

- Promover la cohesión e integración social a través del espacio público que emerge del sistema de PA+ECAs -que funcionan a modo de nodos- interconectados por redes/trama, que construye y pone en valor una poli centralidad que hoy no existe, así como también potencia el espacio público como elemento articulador entre los diferentes nodos para la cohesión y la integración social, que permiten utilizar diferentes modalidades de movilidad.

- Mejorar los servicios básicos en sus distintas escalas, planteando un abordaje innovador en materia ambiental, al incorporar y visibilizar el agua como parte de la urbanización; generando interrelaciones entre vecinos y cursos de los arroyos a través de parques públicos que los incluyen.

- Se propone el saneamiento ambiental natural a través de la fitodepuración en la cuenca alta, así como el tratamiento y limpieza del cauce, promoviendo la formación de cooperativas como actividad económica tendiente al reciclaje y tratamiento de los residuos sólidos que se presentan como una problemática de contaminación ambiental importante del barrio.

- Mejorar el nivel de accesibilidad, en distintas escalas. A nivel interbarrial y entre subcuencas, estableciendo un sistema de movimiento principal sobre las arterias estructurantes del sector que se prolongan del trazado del Casco Fundacional de la Ciudad y otro secundario, que articula los distintos PA+ECAs, con un sistema de bicisendas para la movilidad interna y el movimiento lento al interior del sistema principal que definen las macro manzanas del trazado. Asimismo, a escala del Parque, mediante senderos que definen distintas bandas programáticas se facilita el acceso al equipamiento comunitario y al espacio público propuesto.

- Adaptar la materialidad de calles, veredas, ramblas u otros espacios públicos con materiales y diseños que procuren el mayor grado de infiltración posible e incrementar la arborización.

- Fortalecer el capital humano, dado que los ECAs plantean actividades orientadas a estimular y fortalecer la educación ambiental de modo integral a través de talleres, muestras, que incluyen el reciclaje, la recuperación y la reutilización de residuos; exploración y conocimiento de los servicios ambientales que procura la naturaleza; a la vez que promueven actividades productivas de auto cultivo (huertas) y mercado comunitario (feria barrial).

${ }^{4}$ Escala Urbanística y Arquitectónica: Distinción Bienal de Arquitectura Argentina (BIA-AR) 2018. Buenos Aires (Argentina). 


\section{SIIU}

barcelona|santiago de chile junio 2019
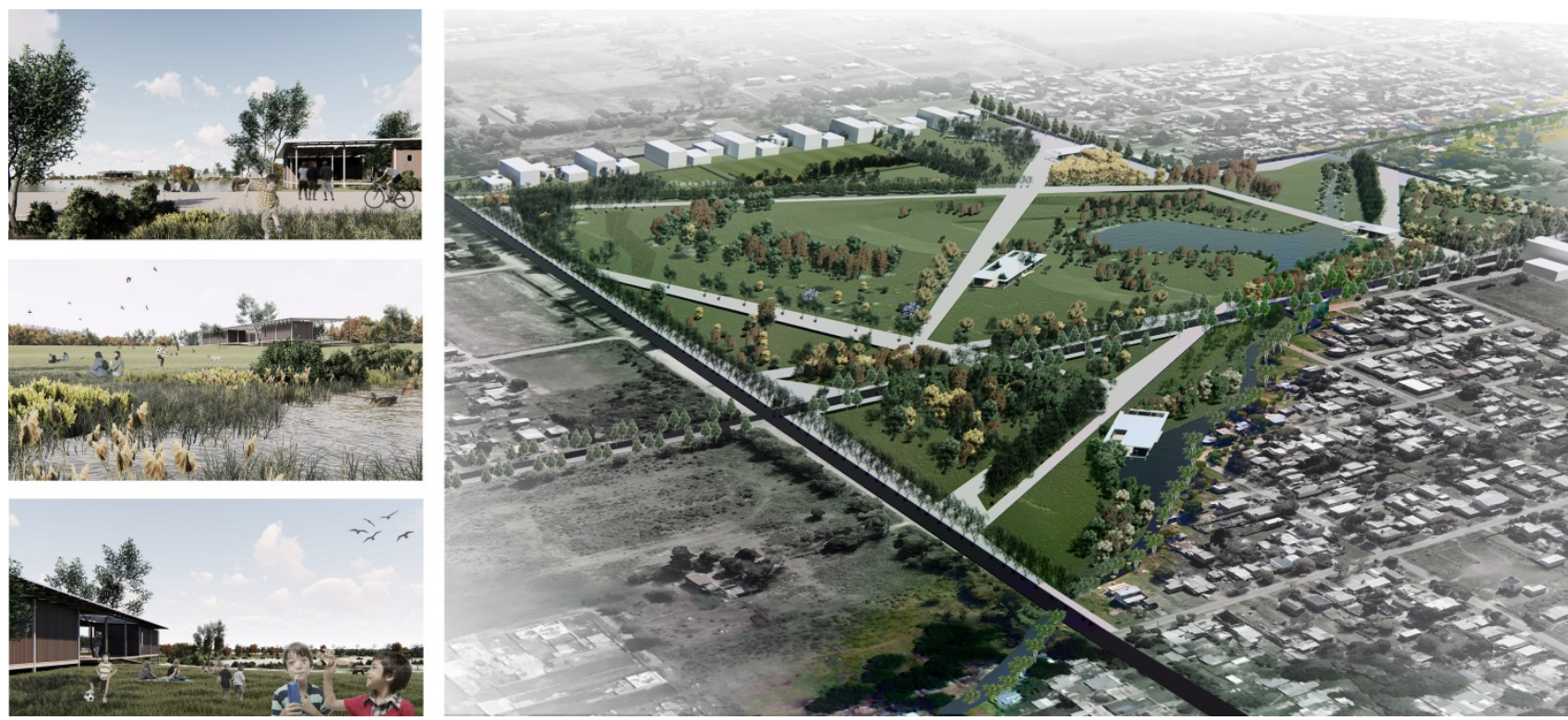

Figura 5. Parques del Agua PA. Fuente: Elaboración propia

A escala Arquitectónica, se propone:

- Rever el Código de Edificación y/o Construcción asociado a la zonificación de riesgo y con la participación de la población de cada zona para orientar la construcción de los edificios, en cuanto a los aspectos estructurales, hidráulicos, de material y sellados. Será obligatorio construir un nivel superior por encima de la crecida probable.

- Rever el factor de ocupación del suelo (FOS) así como el factor de ocupación total (FOT) en relación al parcelamiento, su potencialidad y el logro del hidrograma cero a nivel de cada parcela.

- Incorporar en el sistema edilicio existente y futuro reguladores/retardadores del agua de lluvia.

- Generar Equipamientos Comunitarios Ambientales (ECAs), que doten al sistema de Parques de Agua de una multifuncionalidad programática, que sirvan como puntos de referencia de la población barrial y contribuyan a la construcción de resiliencia comunitaria.
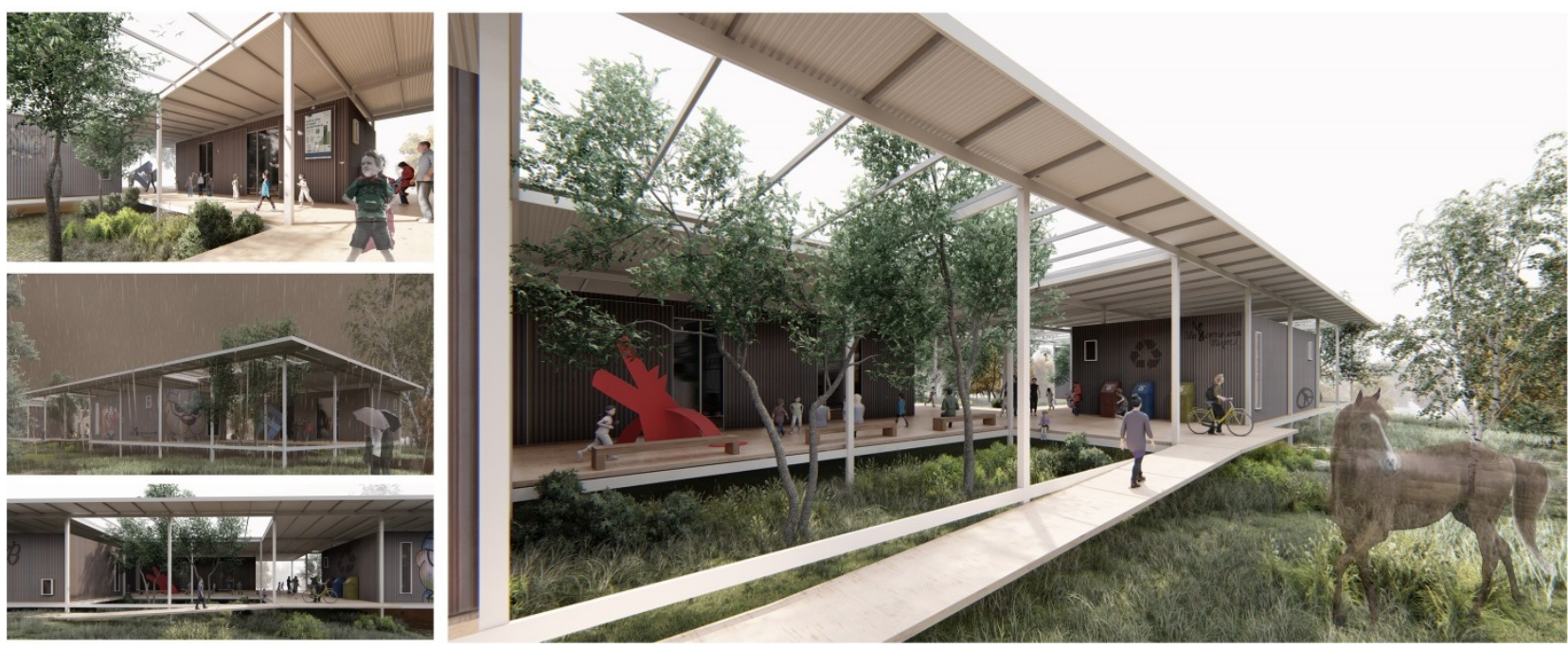

Figura $N^{\circ}$ 6. Equipamientos Comunitarios Ambientales ECAs. Elaboración propia 


\section{Reflexiones finales}

Los problemas de la interescalaridad para enfrentar el riesgo hídrico por inundaciones urbanas y los problemas asociados a la cuestión ambiental afecta a toda la población en distintos grados. Esta presentación ha sido solo un recorte en el territorio que debería replicarse en todas las cuencas y los ARTEH y en la adaptación de la urbanización en general.

Mejorar la resiliencia urbana está en la naturaleza de las estrategias formuladas en las diferentes escalas de abordaje de las propuestas.

"Construir resiliencia", es el eje central del proyecto de investigación en desarrollo.

\section{BIBLIOGRAFÍA}

BECK, U. (2006). La sociedad del riesgo global. Madrid: Siglo XXI de España Editores.

CIIFEN. (2013). Centro Internacional para la Investigación del Fenómeno del Niño. http://www.ciifen.org

CIPPONERI, M., SALVIOLI, M. L., LARRIVEY, G., AFRANCHI, A. V. y COLLI, G. A. (2014). Vulnerabilidad de la Población de la Ciudad de La Plata (Argentina) a Precipitaciones Extraordinarias. Revista Aqua-LAC. UNESCO (Montevideo, Uruguay), Vol. 6.

CIUT. (2014). Proyecto Tierras 1-SIG. Subproyecto Hábitat Informal en el Gran La Plata. Convenio Secretaria Nacional de Acceso al Hábitat (Argentina). CIUT. FAU. UNLP.

FUNTOWICZ, S. y RAVETZ, J. (1993). Riesgo Global, Incertidumbre e Ignorancia. En Epistemología Política. Ciencia con la gente. Buenos Aires: CEAL.

FUNTOWICZ, S. (1994). Epistemología Política. Ciencia con la gente. Conferencia desarrollada en FLACSO 31-5-94. En: NATENZON, E (Comp.). Serie Documentos e Informes de Investigación Nº178. Buenos Aires: FLACSO.

GUIMARAES PEREIRA, A. y FUNTOWICZ, S. (2015). Science, Philosophy and Sustainability: The end of de Cartesian Dream. Routedge series Explorations in Sustainability and Governance. New York: Routedge.

HERZER, H. y GUREVICH, R. (1996). Construyendo el riesgo ambiental en la ciudad. En Desastres y Sociedad No7. Revista semestral de la Red de Estudios Sociales en prevención de desastres en América Latina (Panamá).

LAVELL, A. (1997). Viviendo en riesgo: comunidades vulnerables y prevención de desastres en América Latina. Buenos Aires: Red de Estudios Sociales en Prevención de Desastres en América Latina. LA RED.LÓPEZ, Isabel (2018). Compiladora. Capítulo 3. Riesgo de Inundación en zonas urbanas y estrategias de mitigación y adaptación. Aspectos teórico-metodológicos y propositivos. Autores: LÓPEZ, I. y ETULAIN, J. En el libro: Inundaciones por lluvia en el Sur de la Región Metropolitana de Buenos Aires. Riesgos y Estrategias en La Plata, Berisso y Ensenada. Buenos Aires: Editorial Espacio.

LÓPEZ, I. y otros. Concurso Banco de Desarrollo para América Latina (CAF) 2018. Distinguido en el Área Prácticas Académicas, eje sustentabilidad física y social por la Bienal de Arquitectura Argentina (Buenos Aires).

NACIONES UNIDAS (2009). Terminología sobre Reducción de Riesgo de desastres. En Estrategias para la Reducción de Desastres de las Naciones (UNISDR). Naciones Unidas, Suiza. http://www.unisdr.org/files/7817 UNISDRTerminologySpanish.pdf 
NATENZON, C. (1995). Catástrofes naturales, riesgo e incertidumbre. Serie de Documentos e Informes de Investigación No197.Buenos Aires: FLACSO.

RIBERA MASGRAU, L. (2004). Los Mapas de Riesgo de Inundaciones: representación de la vulnerabilidad y aportaciones de las innovaciones tecnológicas. En Documents d'anàlisi geográfica Vol.43. Barcelona: Universitat Autònoma de Barcelona y Universitat de Girona.

ROMANAZZI, P. y URBIZTONDO, J. (2007). Estudios Hidrológicos-Hidráulicos-Ambientales en la Cuenca del Arroyo del Gato. Informe Final. Laboratorio de Hidrología, Área Departamental Hidráulica, Facultad de Ingeniería, Universidad Nacional de La Plata (Argentina).

SAUTU, R. (2003) Todo es teoría. Buenos Aires: Ediciones Lumière.

TUCCI, C. E. M. (2007). Gestión de Inundaciones Urbanas. Secretaría de la Organización Meteorológica Mundial. Comité Permanente de los Congresos Nacionales del Agua. Argentina.

VELTZ, J. y KRELLENBERG, K. (2016). Vulnerabilidad frente al cambio climático en la Región Metropolitana de Santiago de Chile: posiciones teóricas versus evidencias empíricas. En Revista EURE, Vol. 42. Pp. 251272. Santiago de Chile (Chile).

WOLANSKY, S. y CORZO, H. (2003). Las Inundaciones en Santa Fe - Desastres Naturales y Mitigación del Riesgo. Santa Fe: Centro Publicaciones UNL. (Argentina). 